\title{
The Impact of Icariside II on Human Prostate Cancer Cell Proliferation, Mobility, and Autophagy via PI3K-AKT-mTOR Signaling Pathway
}

This article was published in the following Dove Press journal: Drug Design, Development and Therapy

\section{Shuang Li* \\ Yunlu Zhan (1D* \\ Yingwei Xie* \\ Yonghui Wang \\ Yuexin Liu (D)}

Department of Urology, Beijing Tongren Hospital, Capital Medical University, Beijing 100730, People's Republic of China

*These authors contributed equally to this work
Correspondence: Yuexin Liu

Department of Urology, Beijing Tongren Hospital, Capital Medical University, No.

I, Dongjiaomin Lane, Dongcheng District, Beijing 100730, People's Republic of China

Tel +86 I370I076733

Fax +86 10-58268536

Email doctorlyx@I26.com
Introduction: The flavonol glycoside icariside II (ICA II) has been shown to exhibit a range of anti-tumor properties. Herein, we evaluated the impact of ICA II on human prostate cancer cell proliferation, motility, and autophagy, and we further evaluated the molecular mechanisms underlying these effects.

Methods: We treated DU145 human prostate cancer cells with a range of ICA II doses and then assessed their proliferation via CCK-8 assay, while flow cytometry was used to monitor apoptosis and cell cycle progression. We further utilized wound healing and transwell assays to probe the impact of ICA II on migration and invasion, and assessed autophagy via laser confocal fluorescence microscopy. Western blotting was further utilized to measure LC3-II/I, Beclin-1, P70S6K, PI3K, AKT, mTOR, phospho-AKT, phospho-mTOR, and phosphoP70S6K levels, with qRT-PCR being used to evaluate the expression of specific genes at the mRNA level.

Results: We found that ICA II was capable of mediating the dose- and time-dependent suppression of DU145 cell proliferation, causing these cells to enter a state of cell cycle arrest and apoptosis. We further determined that ICA II treatment was associated with significant impairment of prostate cancer cell migration and invasion, whereas autophagy was enhanced in treated cells relative to untreated controls.

Conclusion: Our results indicate that ICA II treatment is capable of suppressing human prostate tumor cell proliferation and migration while enhancing autophagy via modulating the PI3K-AKT-mTOR signaling pathway. As such, ICA II may be an ideal candidate drug for the treatment of prostate cancer.

Keywords: icariside II, prostate cancer, PI3K-AKT-mTOR, autophagy, apoptosis

\section{Introduction}

Prostate cancer (PC) remains one of the leading causes of cancer and death among men. ${ }^{1}$ Radical prostatectomy is the main method used to treat localized prostate cancer, ${ }^{2}$ while androgen deprivation therapy (ADT) is the most important treatment in patients with advanced-staged PC. ${ }^{3}$ While initially efficacious in those with androgen-sensitive PC, most patients eventually exhibit ADT resistance such that their disease is reclassified as castration-resistant PC (CRPC) and has a poor prognosis. $^{2,3}$ As such, it is vital that novel treatments for CRPC be identified.

Many natural products from traditional medicinal herbs have been leveraged to treat cancer in recent years. The flavanol glycoside icariside II (ICA II) is a primary compound isolated from the traditional Chinese medicinal compound Herba 
epimedii. ${ }^{4,5}$ ICA II has been found to exhibit a diverse array of biological and pharmacological activities, serving to combat cancer, sexual dysfunction, and osteoporosis in multiple studies. ${ }^{4,5}$ ICA II can inhibit the COX-2/PGE 2 pathway and induce mitochondria-dependent apoptosis in PC cells. ${ }^{6}$ ICA II is further reported to exhibit anticancer activity against many human cancer cell lines in vitro and in vivo, with such activity being related to the ability of this compound to impact apoptosis and cell cycle progression, as well as the JAK2-STAT3, MAPK-ERK, and $\beta$ Catenin signaling pathways. ${ }^{6}$

Autophagy is a key catabolic process in eukaryotic cells. ${ }^{7}$ The role of autophagy in cancer is complex. Several studies have reported that autophagy can both suppress tumor growth by inhibiting the accumulation of damaged organelles and misfolded protein aggregates, while also promoting the survival and consequent growth of established tumors. ${ }^{8,9}$ Recently, autophagy has been highlighted as a potentially viable therapeutic target for the treatments of CRPC., ${ }^{3,7}$ The phosphatidylinositol 3-kinase-protein kinase B-mammalian target of rapamycin (PI3K-AKT-mTOR) signaling pathway is an essential regulator of activities such as cellular motility, proliferation, and autophagy. ${ }^{8-10}$ The present study was therefore designed with the goal of evaluating the impact of ICA II on human PC cell proliferation, migration, and autophagy and the mechanisms underlying such activity.

\section{Materials and Methods}

\section{Materials}

Dulbecco's Modified Eagle Medium (DMEM), fetal bovine serum (FBS), and penicillin/streptomycin were obtained from Gibco (Life Technologies, NY, USA). Phosphate buffered saline (PBS), protease and phosphatase inhibitor cocktails, bovine serum albumin (BSA), Radio-Immunoprecipitation Assay (RIPA) lysis buffer, stripping buffer, propidium iodide (PI), and thioglycollate were from Sigma Aldrich (St. Louis, MO, USA). An annexin V-FITC-base apoptosis detection kit, a Cell Counting Kit-8 (CCK-8), and Transwell chambers (with Matrigel pre-coating) were from BD Biosciences (San Jose, CA, USA). Antibodies specific for microtubuleassociated protein 1A/1B-light chain 3 (LC3), Beclin1, P70S6K, PI3K, AKT, mTOR, phospho-AKT, phosphomTOR, and phospho-P70S6K were from Cell Signaling (Santa Cruz, CA, USA).

\section{Ethics Statement}

DU145 cells were obtained from the Cell Bank of Chinese Academy of Sciences (Shanghai, China). All experimental procedures were carried out in accordance with the guidelines of the Chinese Care and Use legislation, and were approved by the Animal Ethics Committee of Beijing Tongren Hospital, Capital Medical University.

\section{Cell Culture}

DU145 cells were cultured in DMEM containing 10\% FBS and penicillin/streptomycin at $37^{\circ} \mathrm{C}$ in a $5 \% \mathrm{CO} 2$ incubator.

\section{Cell Proliferation Assay}

A CCK-8 assay was used to assess the impact of ICA II on DU145 cell proliferative activity. Briefly, DU145 cells were added to a 96-well plate and were treated for 12,24 , or 48 h using $0,10,20,40$, or $80 \mu \mathrm{M}$ ICA II. A CCK- 8 kit was then used based on provided directions, with absorbance (OD) at $450 \mathrm{~nm}$ being evaluated via Multiclan EX plate reader (Thermo Fisher Scientific, MA, USA). Proliferation $(\%)=(\mathrm{OD}$ of treated cells $-\mathrm{OD}$ of blank sample $) /(\mathrm{OD}$ of control sample - OD of blank sample) $\times 100$.

\section{Assessment of Cell Cycle Progression}

The impact of ICA II on cell cycle progression in DU145 cells was assessed using a PI kit. Briefly, cells were treated for $48 \mathrm{~h}$ using 0,20 , or $40 \mu \mathrm{M}$ ICA II in 6-well plates, after which they were fixed overnight using $70 \%$ ethanol at $-20^{\circ}$ C. Cells were then washed two times using PBS prior to being stained with PI. A flow cytometer was then used to evaluate these cells, and FlowJo was used for data analysis.

\section{Assessment of Cellular Apoptosis}

Apoptosis was assessed using an Annexin V-FITC/PI kit. Briefly, we treated DU145 cells for 48 h using 0,20 , or 40 $\mu \mathrm{M}$ ICA II for $48 \mathrm{~h}$ in 6-well plates, after which the cells were washed two times with chilled PBS before being resuspended in a $100 \mathrm{uL}$ volume of binding buffer. Next, $5 \mathrm{uL}$ each of PI and Annexin V-FITC was added to each sample, and cells were stained for 10 mins protected from light. An additional $400 \mathrm{uL}$ of binding buffer was then added to each sample prior to analysis using a flow cytometer within $1 \mathrm{hr}$. FlowJo was used for data analysis.

\section{Wound Healing Assay}

DU145 cell migration was assessed via wound healing assay. Briefly, we treated Du145 cells in 6-well plates for 
$48 \mathrm{~h}$ with 0,20 , or $40 \mu \mathrm{M}$ ICA II. A micropipette tip was then used to generate a wound in the cell monolayer, with PBS being used to remove detached cells. Serum-free media was then added, and wound closure was assessed via light microscopy at appropriate time points.

\section{Invasion Assay}

DU145 cell invasion was assessed with a Transwell assay system. Briefly, DU145 cells were treated for $48 \mathrm{~h}$ using 0, 20 , or $40 \mu \mathrm{M}$ ICA II in 6-well plates. Cells were resuspended in serum-free DMEM and added to the upper chamber of a Matrigel-coated Transwell insert (8um pore size), with DMEM containing 15\% FBS being added to the lower well. After incubation for $24 \mathrm{~h}$, invasive cells were fixed using 4\% paraformaldehyde, stained with crystal violet, and imaged via microscopy.

\section{GFP-LC3 Plasmid Transient Transfection}

Pre-prepared DU145 cells were added to 6-well plates for $24 \mathrm{~h}$ and were transfected transiently with the GFP-LC3 plasmid using Lipofectamine 2000 (Invitrogen, Shanghai, China) based on provided directions. After an additional $24 \mathrm{~h}$, cells were used to assess autophagy by evaluating these GFP-LC3 overexpressing cells via confocal laser scanning microscope (Carl Zeiss, Shanghai, China). 6-diamidino-2-phenylindole (DAPI) was used to stain the nucleus and the green spots were known as autophagosomes.

\section{Western Blotting}

DU145 cells were collected, rinsed with cold PBS, and lysed with RIPA buffer that contained protease and phosphatase inhibitors. Cellular proteins were then separated via SDS-PAGE, transferred to PVDF membranes, and these blots were blocked using 5\% BSA for $1 \mathrm{hr}$. Blots were next probed overnight at $4^{\circ} \mathrm{C}$ using primary antiLC3, Beclin1, P70S6K, PI3K, AKT, mTOR, p-AKT, $\mathrm{p}-\mathrm{mTOR}$, and $\mathrm{p}-\mathrm{P} 70 \mathrm{~S} 6 \mathrm{~K}$. They were then rinsed using TBST, and were probed for $1 \mathrm{~h}$ using HRP-linked secondary antibodies, after which an ECL Advance Detection System was utilized for protein detection.

\section{qRT-PCR}

Gene expression in DU145 cells was assessed via qRTPCR. Primers specific for LC3, Beclin-1, PI3K, AKT, mTOR, and GAPDH were constructed based upon cDNA sequences from the NCBI database. ${ }^{11}$ RNA was extracted from cells using the NucleoSpin RNA isolation kit according to the manufacturer's instructions. Then, qRTPCR reactions were performed in a $10-\mu \mathrm{L}$ reaction volume with the PCR Core Kit. Gene expression levels were calculated via the $2-\Delta \Delta \mathrm{Ct}$ method. GAPDH was used as an internal control to quantify and normalize these results.

The specific primers used in this study were synthesized by Shanghai Bioengineering Co., Ltd, and were as follows: GAPDH-F: TCAAGAAGGTGGTGAAGCAGG, GAPDH-R: GCGTCAAAGGTGGAGGAGTG; Becliin1-F: GGTTGCGGTTTTTCTGGGAC, Beclin-1-R: ACG TGTCTCGCCTTTCTCAA; mTOR-F: CCTCCATCCAC CTCATCA, mTOR-R: GACGCCAAGACACAGTAG LC3-F: TTCTTCCTGCTGGTGAAC, LC3-R: GAGGCG TAGACCATATAGAG; P70S6K-F: CCATGAAGGTGC TTAAAAAGGCA, P70S6K-R: TTCCACCAGTCTGAA AGGCA.

\section{Statistical Analysis}

Statistical testing was conducted using SPSS 22.0 (SPSS Inc., IL, USA) by researchers not involved in conducting this study. Data were compared via one-way ANOVAs, with $\mathrm{P}<0.05$ as the significance threshold.

\section{Results}

\section{ICA II Disrupts the Proliferation of DUI45 Cells in a Dose- and Time-Dependent Manner}

We began by assessing the proliferation of DU145 cells following treatment with a range of ICA II concentrations $(0,10,20,40$, and $80 \mu \mathrm{M})$ for 12,24 , or $48 \mathrm{~h}$. We found that ICA II treatment was associated with significant reductions in the proliferation of these $\mathrm{PC}$ cells $(\mathrm{P}<0.01)$, with the magnitude of this suppression being both timeand dose-dependent (Figure 1).

\section{ICA II Alters Cell Cycle Progression in DUI45 Cells}

We next evaluated DU145 cell cycle progression after treatment a range of ICA II doses $(0,20$, or $40 \mu \mathrm{M})$. ICA II-treated cells exhibited a higher frequency of cells in the G1 phase relative to untreated control cells $(\mathrm{P}<0.01)$, whereas ICA II treatment was associated with a significant reduction in the frequency of cells in the $\mathrm{S}$ phase of the cell cycle $(\mathrm{P}<0.01)$. These results thus suggested that ICA II can induce G1 phase arrest in DU145 cells (Figure 2). 


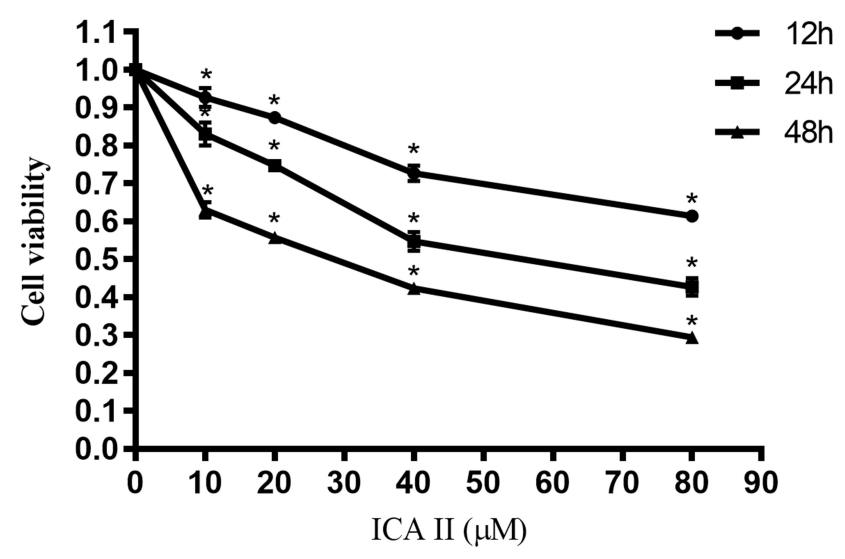

Figure I The impact of ICA II treatment on DUI 45 cell viability. DUI45 cells were treated with ICA II for 12,24 , or $48 \mathrm{~h}$. Results are presented as the mean \pm SD. *P $<0.01$, vs control.

\section{ICA II Induces DUI45 Cellular Apoptosis}

We next used Annexin V/PI staining to assess rates of apoptosis in DU145 cells following a $48 \mathrm{hr}$ treatment with a range of ICA II doses $(0,20$, and $40 \mu \mathrm{M})$. We found that ICA II treatment was associated with significantly higher rates of apoptosis in treated cells relative to untreated cells $(\mathrm{P}<0.01)$, suggesting that this compound is capable of inducing the apoptotic death of these PC cells (Figure 3 ).

\section{ICA II Impacts the Migratory Activity of DUI 45 Cells}

Next, a wound-healing assay was utilized as a means of assessing DU145 cell migration following a $48 \mathrm{~h}$ treatment with a range of ICA II concentrations $(0,20$, and $40 \mu \mathrm{M})$. We observed significantly reduced wound healing in ICA II-treated cell samples relative to control untreated wells $(\mathrm{P}<0.01)$, thus suggesting that ICA II can inhibit DU145 cell migration (Figure 4).

\section{ICA II Suppresses the Invasive Activity of DUI45 Cells}

DU145 cell invasive activity was next assessed via Transwell assay following treatment with a range of ICA II doses $(0,20$, and $40 \mu \mathrm{M})$ for $48 \mathrm{~h}$. We observed

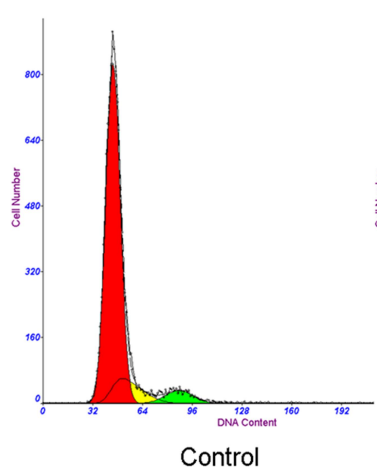

Control

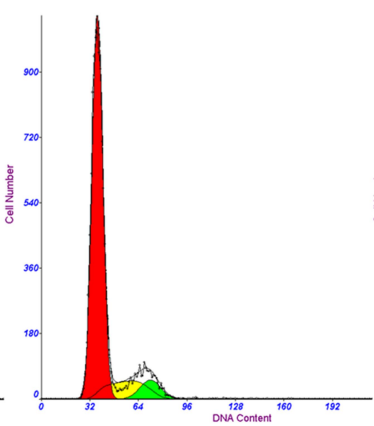

20

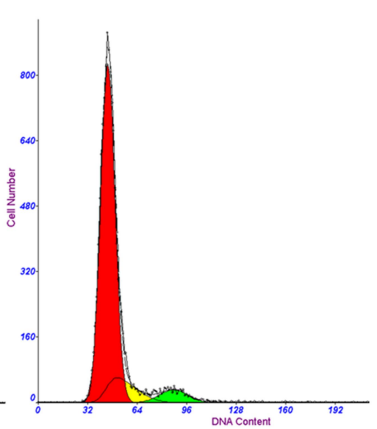

40

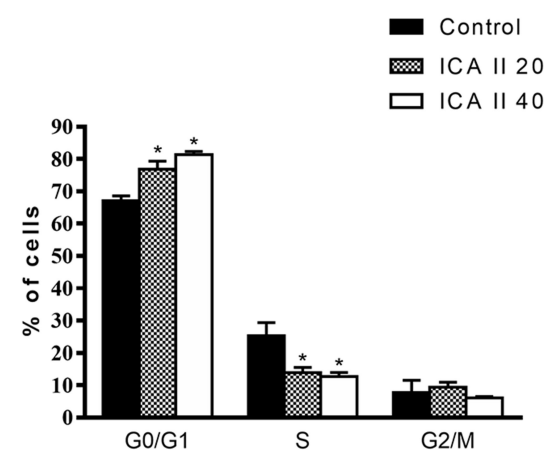

Figure 2 The impact of ICAll on DUI 45 cell cycle distribution. Following a $48 \mathrm{~h}$ treatment with ICA II, DUI 45 cells were assessed via flow cytometry. Percentages of cells in different cell cycle phases are also shown. $* \mathrm{P}<0.01$, vs control.

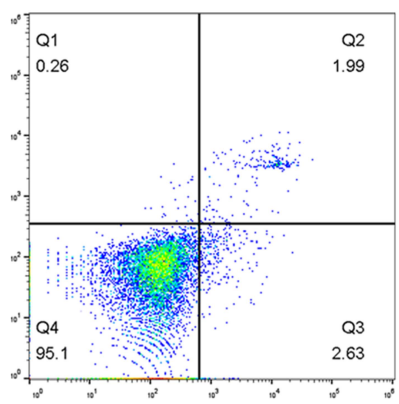

Control

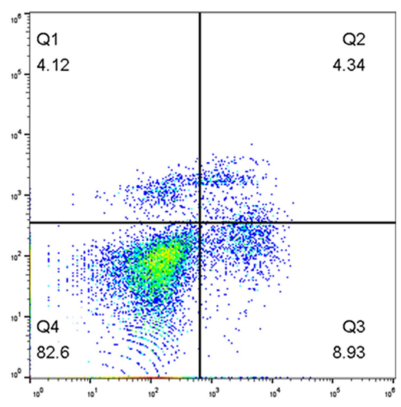

20

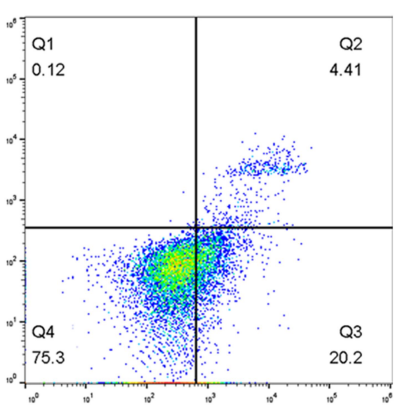

40

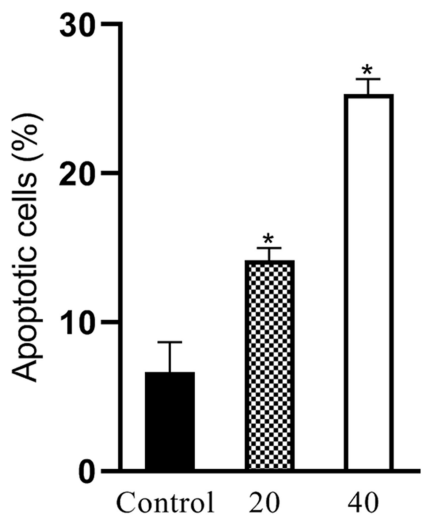

Figure 3 The impact of ICAll on DUI 45 cell apoptosis. Cells were treated for $48 \mathrm{~h}$ with ICA II (0, 20, or $40 \mu \mathrm{M})$, after which flow cytometry was used to assess apoptosis. Percentages of apoptotic cells are shown on the right. *P $<0.01$, vs control. 


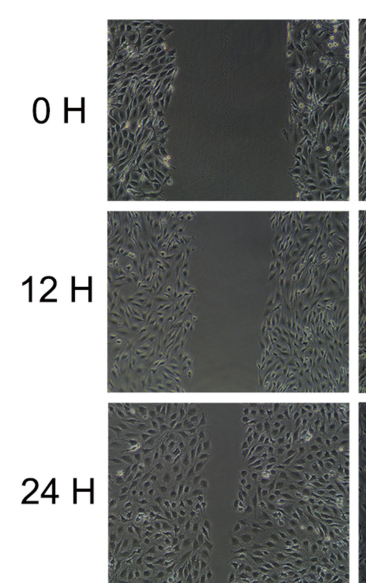

Control
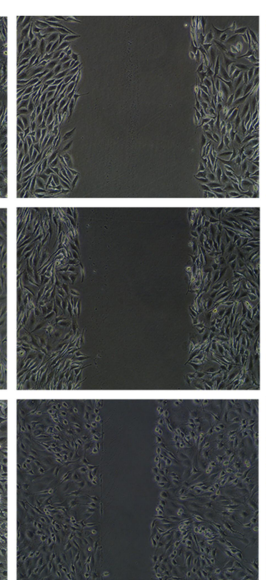

20
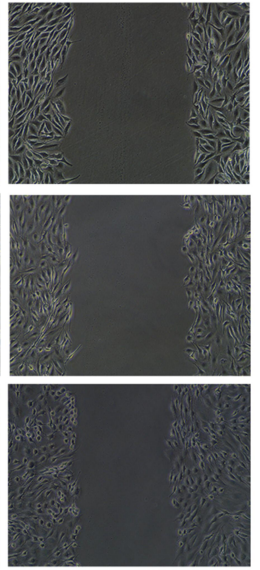

40

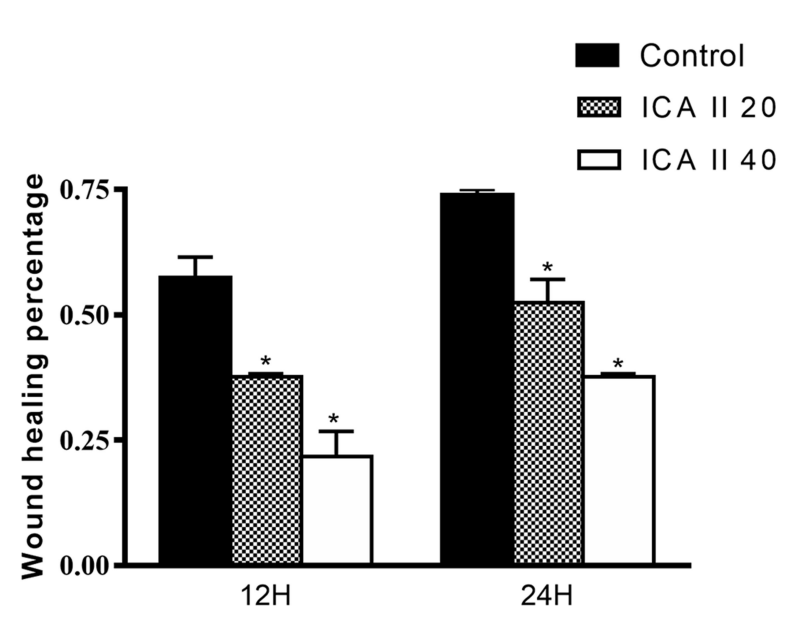

Figure 4 The impact of ICA II treatment on DUI 45 cell migratory activity. DUI45 monolayers were wounded using a pipette tip, with wound healing percentages shown. The ability of ICA II to inhibit DUI 45 cell migration was quantified. $* \mathrm{P}<0.0$ I, vs control.

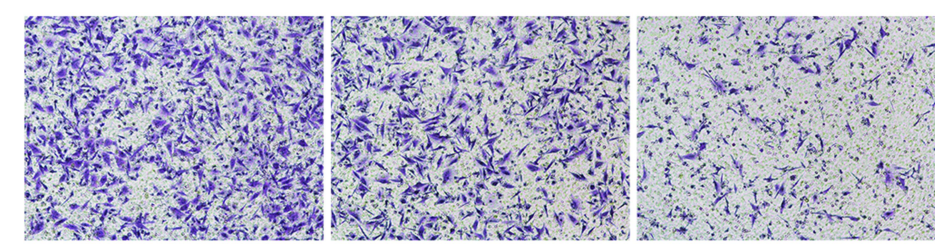

Control

20

40

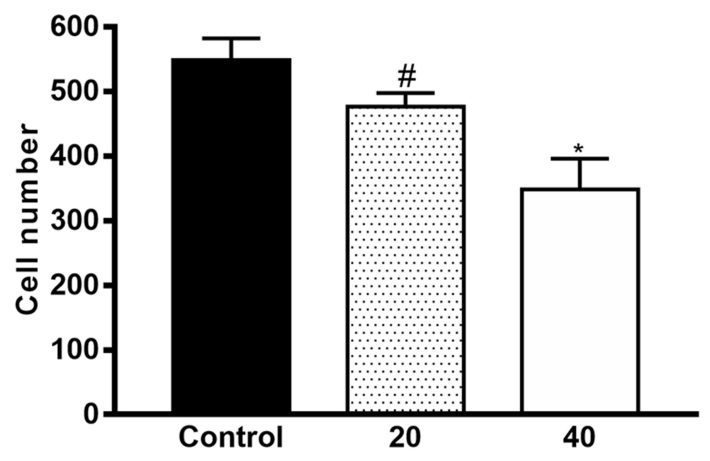

Figure 5 The impact of ICAll on the invasion of DUI 45 cells through Matrigel-coated Transwell filters. The ability of ICA II to inhibit DUI 45 cell invasion was quantified. ${ }^{\#} \mathrm{P}<0.05$, vs control; $* \mathrm{P}<0.01$, vs control.

significant reductions in the number of invasive cells in ICA II-treated wells relative to control untreated wells $(\mathrm{P}<0.01)$, indicating that ICA II is an inhibitor of DU145 cell invasion (Figure 5).

\section{ICA II Induces Autophagic Activity in DUI45 Cells}

We next employed a laser confocal fluorescence microscopy approach to evaluate the impact of ICA II treatment $(0,20$, and $40 \mu \mathrm{M})$ on autophagic activity in DU145 cells. We observed enhanced autophagosome formation in ICA II-treated DU145 cells relative to control untreated cells $(\mathrm{P}<0.01)$, suggesting that ICA II is capable of promoting autophagy within these PC cells (Figure 6).

\section{The Impact of ICA II on the PI3K-Akt- mTOR Signaling Pathway and Autophagy-Related Protein Expression in Prostate Cancer Cells}

The PI3K-Akt-mTOR signaling pathway is related to autophagy, and as such, we assessed the expression of proteins related to this pathway in ICA II-treated DU145 cells. We found that ICA II 40 treatment was linked to a significant increase in the protein-level expression of LC3-II/I and Beclin-1 relative to control samples $(\mathrm{P}<0.01)$, whereas ICA II treatment was associated with reductions in the expression of PI3K, p-AKT $(\mathrm{P}<0.05)$, p-mTOR, and p-P70S6K levels relative to those observed in control samples $(\mathrm{P}<0.01)$. In contrast, total mTOR, AKT, and P70S6K expression levels 


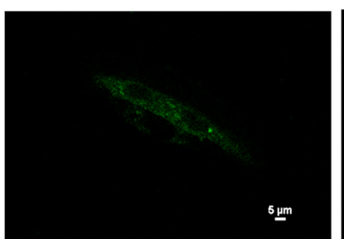

Control

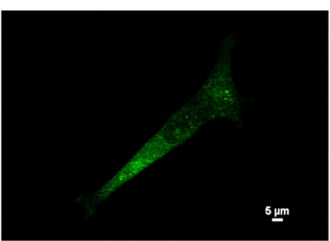

20

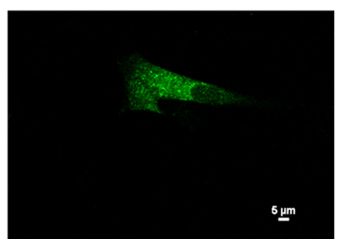

40

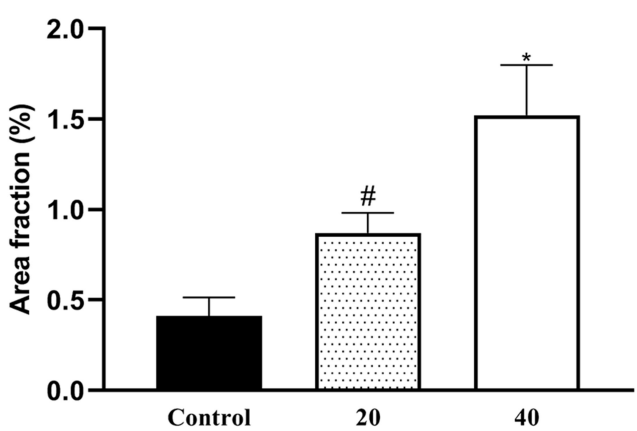

Figure 6 The impact of ICAll on DUI45 cell autophagy. DUI45 cells were transfected using the GFP-LC3 plasmid. The autophagic area as a fraction of the total area in these DUI45 cells was quantified. ${ }^{\#} \mathrm{P}<0.05$, vs control; ${ }^{*} \mathrm{P}<0.0$ I, vs control.

were comparable between ICA II-treated and control group samples $(\mathrm{P}>0.05)$ (Figures 7 and 8 ).

\section{The Impact of ICA II on PI3K-Akt-mTOR} Signaling Pathway- and Autophagy-Related Gene Expression in Prostate Cancer Cells

Finally, we assessed the mRNA level expression of genes related to autophagy and PI3K-Akt-mTOR activity via qRT-PCR in DU145 cells. We observed significant increases in mRNA level expression of LC3 and Beclin1 in ICA II-treated cells relative to controls $(\mathrm{P}<0.01)$, whereas ICA II reduced the expression of PI3K, AKT, and mTOR relative to expression levels in control cells $(\mathrm{P}<0.01)$. We also found that ICA II treatment was associated with a reduction in $\mathrm{P} 70 \mathrm{~S} 6 \mathrm{~K}$ expression relative to control samples $(\mathrm{P}<0.05)$ (Figures 9 and 10$)$.

\section{Discussion}

Cancer remains the second most prevalent cause of death globally, with PC being the most common cancer affecting men. ${ }^{1}$ Between $10 \%$ and $20 \%$ of men with metastatic PC develop CRPC within a 5-year period, and following the onset of such castration resistance the median survival time for these patients is just 14 months. ${ }^{1,3}$ Chemotherapy is the primary treatment for those with CRPC, and entails the administration of an array of natural or synthetic pharmacological compounds that can help to disrupt tumor cell growth or division. ${ }^{3,11}$ Docetaxel is currently employed as a first-line treatment for CRPC, as it has been shown to yield survival advantages to treated patients, but it is associated with negative
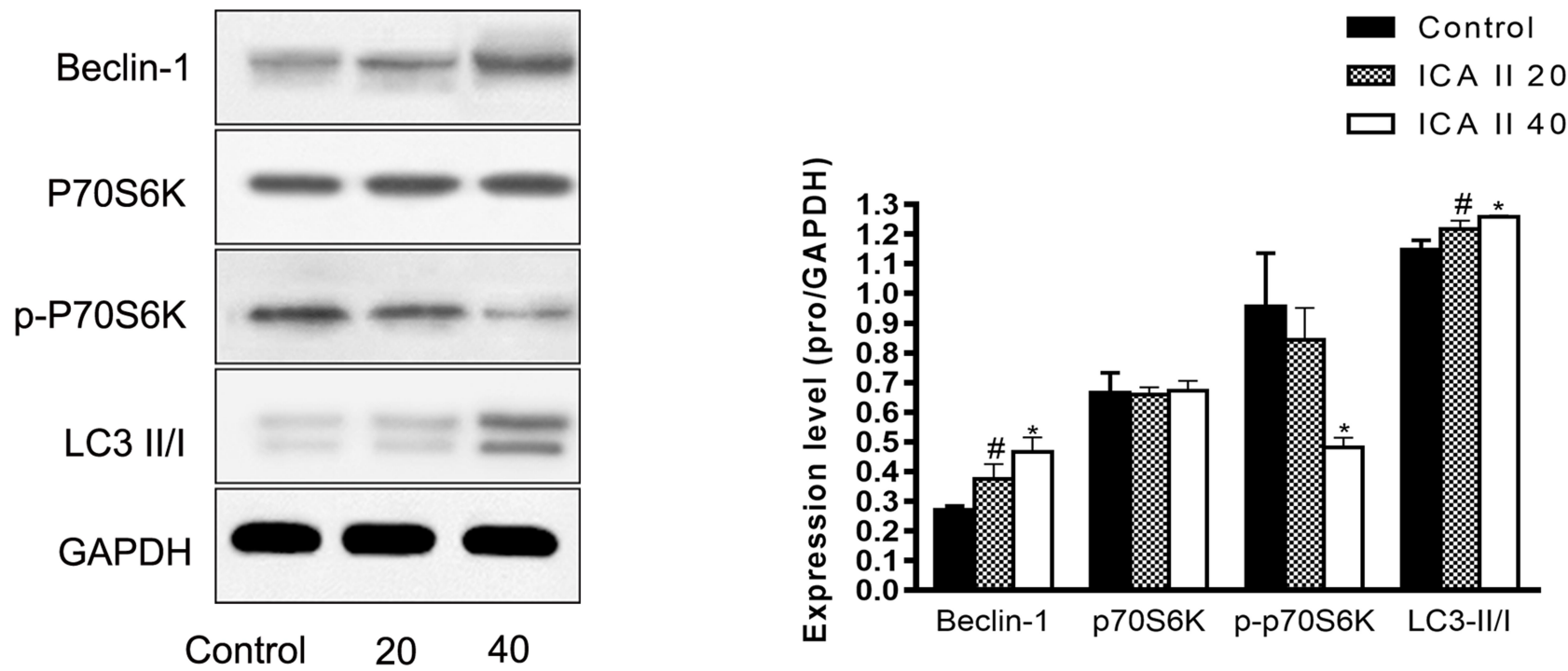

Figure 7 The impact of ICAll on the expression of autophagy-related proteins in DUI45 cells. Levels of Beclin-I, p70S6K, p-P70S6K, and LC3-II/I in DUI45 cells were quantified. *P $<0.0$ I, vs control; ${ }^{\#} \mathrm{P}<0.05$, vs control. 


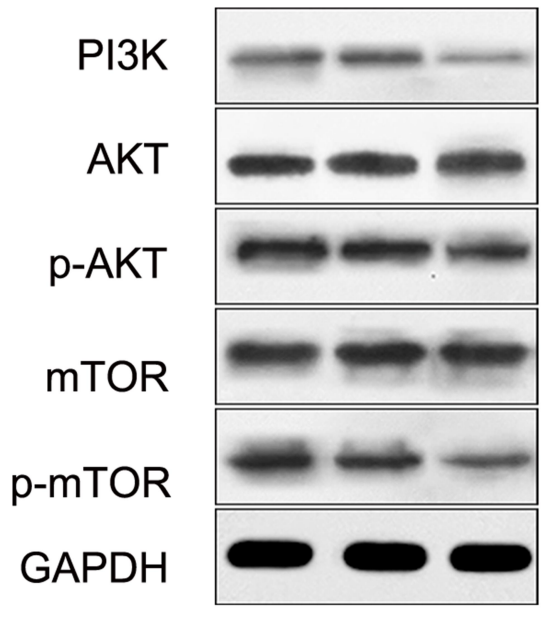

Control $20 \quad 40$

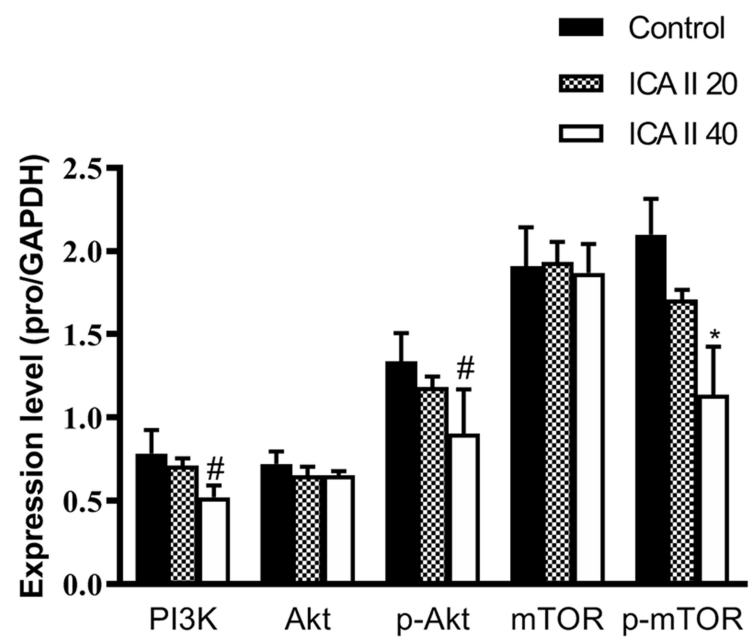

Figure 8 The impact of ICAll on the expression of proteins in the PI3K-AKT-mTOR pathway in DUI45 cells. Levels of PI3K, Akt, p-Akt, mTOR, and p-mTOR in DUI45 cells were quantified. $* \mathrm{P}<0.01$, vs control; ${ }^{\#} \mathrm{P}<0.05$, vs control.

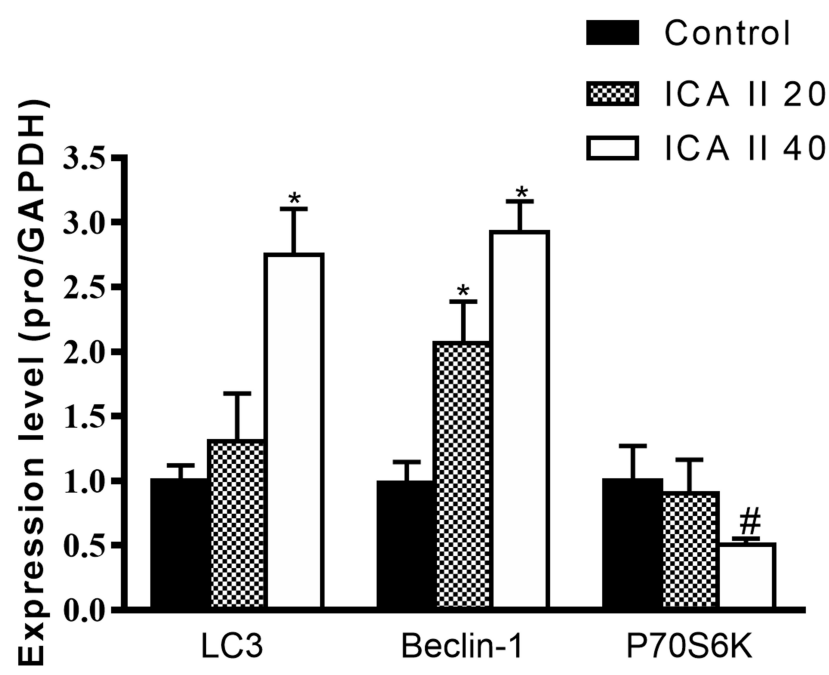

Figure 9 The impact of ICAll on the expression of autophagy-associated genes in DUI45 cells. Beclin-I, P70S6K, and LC3 gene expression in DUI45 cells was quantified. *P $<0.0$ I, vs control; ${ }^{*} \mathrm{P}<0.05$, vs control.

side effects such as diarrhea, hair loss, and myelosuppression. ${ }^{3,7,12}$ DU145 cells are androgenindependent human PC cells and are commonly used as a model for studies of CRPC. Novel therapeutic agents capable of treating CRPC are urgently needed.

Tumor growth and development are associated with increased invasion and dysregulated apoptotic cell death. ${ }^{13,14}$ Several different anti-tumor drugs seek to modulate these activities via disrupting tumor cell proliferation and/or inducing apoptosis. ${ }^{10,15}$ Traditional herbal medicines represent a potentially ideal source of compounds for use in

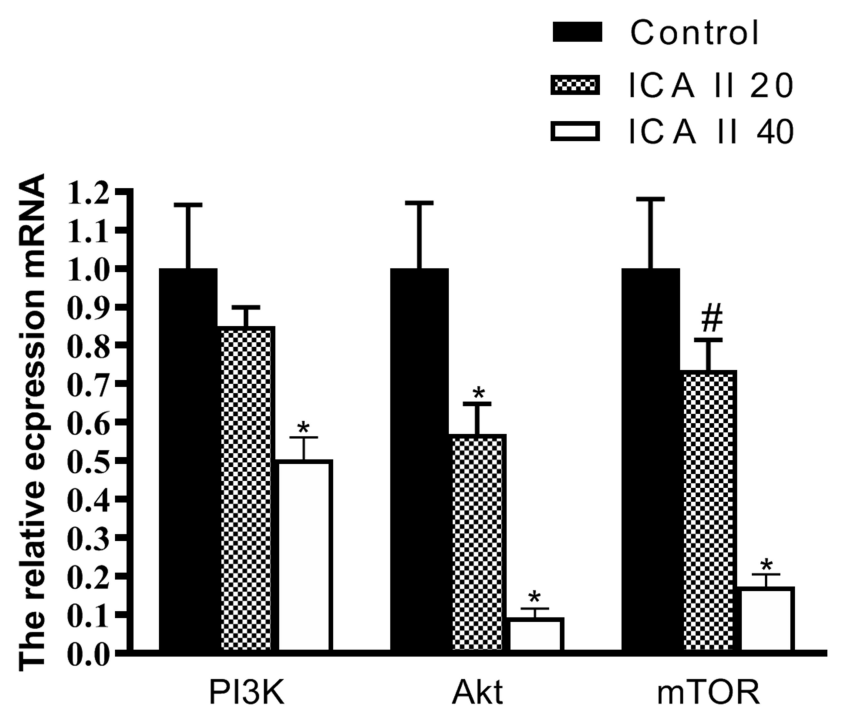

Figure 10 The impact of ICAll on the expression of genes related to PI3K-AKTmTOR signaling in DUI45 cells. PI3K, Akt, and mTOR gene expression in DUI45 cells was quantified. ${ }^{*} \mathrm{P}<0.0$ I, vs control; ${ }^{*} \mathrm{P}<0.05$, vs control.

chemotherapeutic regimens, with drugs such as camptothecin, paclitaxel, and emodin all having been developed from natural plant sources. ${ }^{16-18}$ Previous work has demonstrated that ICA II can mediate anti-tumor activity against hepatic carcinoma, breast cancer, and gastric cancer cells via a range of mechanisms. ${ }^{10,16}$ The P13K-Akt-mTOR signaling axis is a key regulator of protein synthesis within cells stimulated using an array of growth factors, influencing both autophagic activity and survival in a contextdependent manner. ICA II treatment of osteosarcoma cells has recently been shown to be associated with reduced 
PI3k-AKT pathway activation. ${ }^{16}$ Herein, we therefore explored the anti-cancer properties of ICA II by using it to treat DU145 PC cells. We ultimately determined that ICA II was able to reduce the proliferative, migratory, and invasive activity of these cells while inducing excessive autophagy through PI3K-AKT-mTOR signaling pathway-related activity.

Cancer cells are largely defined by the fact that they can undergo unrestrained proliferation while resisting apoptotic death. ${ }^{18}$ Chemotherapeutic drugs function by disrupting these activities, leading cells to be less proliferative and/or more sensitive to apoptotic signaling. ${ }^{19}$ There is previous evidence that ICA II can induce lung cancer cell apoptosis. ${ }^{16}$ The cell cycle defines the basic steps whereby cells are able to replicate and divide, and it is traditionally divided into the $\mathrm{G} 0, \mathrm{G} 1, \mathrm{~S}$, and G2/M phases. ${ }^{73}$ Cell cycle arrest is a primary checkpoint that can help to restrain tumor cell growth, and as such many chemotherapeutic compounds function by inducing such cell cycle arrest within cancerous cells. ${ }^{16,20}$ Herein, we determined that ICA II was capable of significantly reducing DU145 cell proliferation in a dose- and time-dependent fashion, while also inducing G1 phase arrest and apoptosis within these PC cells. As such, the anti-proliferative activity of ICA II may be attributable to its ability to induce cell cycle arrest.

Metastatic progression is a complex, multi-step process wherein tumor cells undergo changes in their migratory, invasive, proliferative, phenotypic, and angiogenic properties that enable them to expand and spread to distant metastatic sites within affected individuals. ${ }^{21-23}$ Both invasion and migration are key drivers of this metastatic process. ${ }^{12}$ A number of studies have explored the ability of ICA II to modulate the invasion and migration of lung, gastric, and esophageal cancer cells. ${ }^{10,16}$ Herein, we determined that ICA II was able to significantly inhibit DU145 $\mathrm{PC}$ cell invasion and migration.

Autophagy serves as a catabolic process in eukaryotic cells and is a vital means of maintaining intracellular homeostasis in physiological and pathological contexts. ${ }^{6,24,25}$ While it can promote cell survival in some cases, in other settings autophagy can trigger apoptotic cell death depending on the intracellular signaling pathways that are engaged in a given cell. ${ }^{6}$ Autophagic cell death is an alternative form of programmed cell death that is distinct from apoptosis and that has been observed in the context of PC. ${ }^{6,26}$ Autophagy is associated with a disruption of apoptotic induction, whereas the caspase activity that is induced during apoptosis can, in turn, disrupt autophagic processes. In some contexts, however, autophagy and related signaling processes can trigger tumor regression and cell death. ${ }^{26}$ The specific mechanisms governing autophagy are highly complex and tightly regulated.

Beclin-1 and LC3 are key proteins that regulate the formation of autophagosomes, thereby controlling the initiation of autophagy. ${ }^{12}$ Beclin-1 interacts with Bcl-2, and was the first mammalian protein shown to be involved in the process of autophagy owing to its key role in inducing autophagosome formation. ${ }^{22,27}$ Beclin-1 overexpression has been reported to inhibit HeLa cell growth in vivo and in vitro, as it induces enhanced autophagic activity and apoptotic death in these tumor cells. The protein LC3 can exist in both solid and lapidated forms known, respectively, as LC3-I and LC3-II. ${ }^{28}$ LC3-II is commonly monitored as a membrane marker for autophagic vacuole formation, and as such, it can be used to gauge autophagy within individual cells. ${ }^{29}$ Indeed, GFP-LC3 is a commonly utilized tool for the assessment of autophagy in cultured cells. ${ }^{30}$ Numerous natural compounds have demonstrated the ability to induce autophagic activity via the regulation of key autophagy-related pathways. ${ }^{15}$ For example, 3-methyladenine (3-MA) can disrupt the formation of autophagosomes via modulating the Beclin-1 pathway. ${ }^{19,30}$ Herein, we determined that ICA II is capable of enhancing autophagic activity within DU145 cells, as evidenced by the increased expression of Beclin-1 and LC3-II therein.

The PI3K-Akt-mTOR signaling pathway is associated with autophagy and controls the differentiation, survival, proliferation, and migration of cells. ${ }^{26}$ Akt functions as the primary mediator of PI3K signaling, with mTOR being a downstream target of this PI3K/Akt signaling pathway. ${ }^{7,9}$ mTOR functionality is primarily governed by the activity of two different complexes (mTORC1 and mTORC2), with mTORC1 kinase activity acting to suppress autophagy and to maintain homeostatic cell growth, proliferation, and survival. PI3K-Akt-mTOR activity has been shown to suppress autophagy, whereas inhibiting this activity results in enhanced autophagic activity. ${ }^{6,31}$ Herein, we found that ICA II was able to induce autophagy within DU145 PC cells, as evidenced by the upregulation of Beclin-1 and downregulation of PI3K, AKT, mTOR, and P70S6K levels within these cells.

PI3k-Akt-mTOR signaling is a key regulator of CRPC cell survival and proliferation, regulating disease progression through crosstalk with other important intracellular signaling pathways associated with survival, differentiation, and autophagy. A number of different drugs that induce autophagy have been shown to mediate specific PI3K-AKT-mTOR pathway downregulation, suggesting that the modulation of this pathway may be a viable approach to inducing targeted tumor cell death. Herein, we found that ICA II was able to inhibit PC cell 
proliferation, invasion, and migration while simultaneously promoting apoptosis and excessive autophagy. The molecular mechanism underlying this phenotype may be associated with disrupted PI3K-AKT-mTOR pathway signaling that ultimately results in the induction of autophagy. This study is, however, limited by the fact that ICA II is a multi-functional anti-tumor compound that can modulate the activity of a range of intracellular targets. As such, our results may only offer insights into one of the several mechanisms whereby ICA II modulates PC cell proliferation and survival. Together, our findings suggest that ICA II may be a viable therapeutic agent for the treatment of CRPC patients.

\section{Conclusions}

Herein, we determined that ICA II can exert a range of antitumor activities by reducing cellular proliferation, impairing migration/invasion, and inducing excessive autophagy via modulating PI3K-AKT-mTOR signaling activity in human PC cells. As such, ICA II may be a viable therapeutic candidate for the treatment of this deadly form of cancer.

\section{Abbreviations}

ICA II, icariside II; PC, prostate cancer; ADT, androgen deprivation therapy; CRPC, castration-resistant PC; PI3KAKT-mTOR, phosphatidylinositol 3-kinase-protein kinase B-mammalian target of rapamycin; FBS, fetal bovine serum; BSA, bovine serum albumin; PI, propidium iodide; LC3, microtubule-associated protein 1A/1B-light chain 3; OD, absorbance; 3-MA, 3-methyladenine.

\section{Funding}

This study was funded by Beijing Municipal Natural Science Foundation (7102033).

\section{Disclosure}

The authors report no conflicts of interest in this work.

\section{References}

1. Siegel RL, Miller KD, Jemal A. Cancer statistics, 2018. CA Cancer J Clin. 2018;68:7-30. doi:10.3322/caac.21442

2. Litwin MS, Tan HJ. The diagnosis and treatment of prostate cancer: a review. JAMA. 2017;317(24):2532-2542. doi:10.1001/jama.2017.7248

3. Mansinho A, Macedo D, Fernandes I, Costa L. Castration-resistant prostate cancer: mechanisms, targets and treatment. Adv Exp Med Biol. 2018;1096:117-133. doi:10.1007/978-3-319-99286-0_7

4. Zhang J, Li S, Zhang S, et al. Effect of icariside II and metformin on penile erectile function, histological structure, mitochondrial autophagy, glucose-lipid metabolism, angiotensin ii and sex hormone in type 2 diabetic rats with erectile dysfunction. J Sex Med. 2020;8 (2):168-177. doi:10.1016/j.esxm.2020.01.006
5. Zhang J, Li S, Li S, et al. Effect of icariside II and metformin on penile erectile function, glucose metabolism, reaction oxygen species, superoxide dismutase, and mitochondrial autophagy in type 2 diabetic rats with erectile dysfunction. Transl Androl Urol. 2020;9 (2):355. doi:10.21037/tau.2020.02.07

6. Chen $\mathrm{M}, \mathrm{Wu} \mathrm{J}$, Luo Q, et al. The anticancer properties of herba epimedii and its main bioactive components icariin and icariside II. Nutrients. 2016;8(9):563. doi:10.3390/nu8090563

7. Sachan R, Kundu A, Jeon Y, et al. Afrocyclamin A, a triterpene saponin, induces apoptosis and autophagic cell death via the PI3K/ Akt/mTOR pathway in human prostate cancer cells. Phytomedicine. 2018;51:139-150. doi:10.1016/j.phymed.2018.10.012

8. Gou XJ, Bai HH, Liu LW, et al. Asiatic acid interferes with invasion and proliferation of breast cancer cells by inhibiting WAVE3 activation through PI3K/AKT signaling pathway. Biomed Res Int. 2020;2020:1874387. doi:10.1155/2020/1874387

9. Yang J, Pi C, Wang G. Inhibition of PI3K/Akt/mTOR pathway by apigenin induces apoptosis and autophagy in hepatocellular carcinoma cells. Biomed Pharmacother. 2018;103:699-707. doi:10.1016/ j.biopha.2018.04.072

10. Kumar S, Ashish J, Won CS, et al. Mammalian Atg8 proteins and the autophagy factor IRGM control mTOR and TFEB at a regulatory node critical for responses to pathogens. Nat Cell Biol. 2020;22 (8):973-985. doi:10.1038/s41556-020-0549-1

11. Zhao X, Chen C, Wei Y, et al. Novel mutations of COL4A3, COL4A4, and COL4A5 genes in Chinese patients with Alport Syndrome using next generation sequence technique. Mol Genet Genomic Med. 2019;7:e653. doi:10.1002/mgg3.653

12. Su CC, Hsieh KL, Liu PL, et al. AICAR induces apoptosis and inhibits migration and invasion in prostate cancer cells through an AMPK/mTOR-dependent pathway. Int J Mol Sci. 2019;20(7):1647. doi:10.3390/ijms20071647

13. Lee HJ, Venkatarame Gowda Saralamma V, Kim SM, et al. Pectolinarigenin induced cell cycle arrest, autophagy, and apoptosis in gastric cancer cell via $\mathrm{PI} 3 \mathrm{~K} / \mathrm{AKT} / \mathrm{mTOR}$ signaling pathway. Nutrients. 2018;10(8):1043. doi:10.3390/nu10081043

14. Fettke H, Kwan EM, Bukczynska P, et al. Prognostic impact of total plasma cell-free DNA concentration in androgen receptor pathway inhibitor-treated metastatic castration-resistant prostate cancer. Eur Urol Focus. 2020. doi:10.1016/j.euf.2020.07.001

15. Huang D, Cui LQ, Ahmed S, et al. An overview of epigenetic agents and natural nutrition products targeting DNA methyltransferase, histone deacetylases and microRNAs. Food Chem Toxicol. 2019;123:574-594. doi:10.1016/j.fct.2018.10.052

16. Khan M, Maryam A, Qazi JI, Ma T. Targeting apoptosis and multiple signaling pathways with icariside ii in cancer cells. Int $J$ Biol Sci. 2015;11(9):1100-1112. doi:10.7150/ijbs.11595

17. Gao P, Huang X, Liao T, et al. Daucosterol induces autophagic-dependent apoptosis in prostate cancer via JNK activation. Biosci Trends. 2019;13 (2):160-167. doi:10.5582/bst.2018.01293

18. Lyu Q, Tou F, Su H, et al. The natural product peiminine represses colorectal carcinoma tumor growth by inducing autophagic cell death. Biochem Biophys Res Commun. 2015;462(1):38-45. doi:10.1016/j.bbrc.2015.04.102

19. Lv Y, Liu Z, Huang J, et al. LncRNA nuclear-enriched abundant transcript 1 regulates hypoxia-evoked apoptosis and autophagy via mediation of microRNA-181b. Mol Cell Biochem. 2020;464(1-2):193-203. doi:10.1007/s11010-019-03660-2

20. Yang J, Lan J, Du H, et al. Icariside II induces cell cycle arrest and differentiation via TLR8/MyD88/p38 pathway in acute myeloid leukemia cells. Eur J Pharmacol. 2019;846:12-22. doi:10.1016/j. ejphar.2018.12.026

21. Fan C, Yang Y, Liu Y, et al. Icariin displays anticancer activity against human esophageal cancer cells via regulating endoplasmic reticulum stress-mediated apoptotic signaling. Sci Rep. 2016;6 (1):21145. doi:10.1038/srep21145 
22. Zhao GS, Gao ZR, Zhang Q, et al. TSSC3 promotes autophagy via inactivating the Src-mediated $\mathrm{PI} 3 \mathrm{~K} / \mathrm{Akt} / \mathrm{mTOR}$ pathway to suppress tumorigenesis and metastasis in osteosarcoma, and predicts a favorable prognosis. J Exp Clin Cancer Res. 2018;37(1):188. doi:10.1186/s13046-018-0856-6

23. Xiong YC, Wang J, Cheng Y, et al. Overexpression of MYBL2 promotes proliferation and migration of non-small-cell lung cancer via upregulating NCAPH. Mol Cell Biochem. 2020;1-9.

24. Chen HE, Lin JF, Tsai TF, et al. Allyl isothiocyanate induces autophagy through the up-regulation of beclin-1 in human prostate cancer cells. Am J Chin Med. 2018:1-19. doi:10.1142/S0192415X18500830.

25. Ahmed M, Lai TH, Zada S, et al. Functional linkage of RKIP to the epithelial to mesenchymal transition and autophagy during the development of prostate cancer. Cancers (Basel). 2018;10(8):273. doi:10.3390/cancers 10080273

26. Liu M, Bamodu OA, Huang WC, et al. 4-Acetylantroquinonol B suppresses autophagic flux and improves cisplatin sensitivity in highly aggressive epithelial cancer through the PI3K/Akt/mTOR/ p70S6K signaling pathway. Toxicol Appl Pharmacol. 2017;325:48-60. doi:10.1016/j.taap.2017.04.003
27. Tsai JH, Hsu LS, Huang HC, et al. 1-(2-Hydroxy-5-methylphenyl)3-phenyl-1,3-propanedione induces G1 cell cycle arrest and autophagy in heLa cervical cancer cells. Int J Mol Sci. 2016;17(8):1274. doi:10.3390/ijms17081274

28. Guan H, Piao H, Qian Z, et al. 2,5-Hexanedione induces autophagic death of VSC4.1 cells via a PI3K/Akt/mTOR pathway. Mol Biosyst. 2017;13(10):1993-2005. doi:10.1039/c7mb00001d

29. Yu Z, Chen Y, Liang C. Eriocalyxin B induces apoptosis and autophagy involving Akt/mammalian target of rapamycin (mTOR) pathway in prostate cancer cells. Med Sci Monit. 2019;25:8534-8543. doi:10.12659/MSM.917333

30. Gioti K, Papachristodoulou A, Benaki D, et al. Silymarin enriched extract (silybum marianum) additive effect on doxorubicin-mediated cytotoxicity in PC-3 prostate cancer cells. Planta Med. 2019;85(11/ 12):997-1007. doi:10.1055/a-0954-6704

31. Rahmani M, Nkwocha J, Hawkins E, et al. Cotargeting BCL-2 and PI3K induces BAX-dependent mitochondrial apoptosis in AML cells. Cancer Res. 2018;78(11):3075-3086. doi:10.1158/0008-5472.CAN17-3024

\section{Publish your work in this journal}

Drug Design, Development and Therapy is an international, peerreviewed open-access journal that spans the spectrum of drug design and development through to clinical applications. Clinical outcomes, patient safety, and programs for the development and effective, safe, and sustained use of medicines are a feature of the journal, which has also been accepted for indexing on PubMed Central. The manuscrip management system is completely online and includes a very quick and fair peer-review system, which is all easy to use. Visit http://www. dovepress.com/testimonials.php to read real quotes from published authors. 